\title{
MicroRNA-376b-3p targets RGS1 mRNA to inhibit proliferation, metastasis, and apoptosis in osteosarcoma
}

\author{
Lei Zhang ${ }^{1}$, Meng Yao ${ }^{1}$, Weikang Ma ${ }^{1}$, Yongqing Jiang ${ }^{1}$, Wenbo Wang ${ }^{2}$ \\ ${ }^{1}$ Department of Orthopedics, the Second Affiliated Hospital of Harbin Medical University, Harbin, China; ${ }^{2}$ Department of Orthopedics, the First \\ Affiliated Hospital of Harbin Medical University, Harbin, China \\ Contributions: (I) Conception and design: L Zhang, W Wang; (II) Administrative support: W Wang; (III) Provision of study materials or patients: L \\ Zhang, M Yao; (IV) Collection and assembly of data: W Ma, Y Jiang; (V) Data analysis and interpretation: L Zhang; (VI) Manuscript writing: All \\ authors; (VII) Final approval of manuscript: All authors. \\ Correspondence to: Wenbo Wang. Department of Orthopedics, the First Affiliated Hospital of Harbin Medical University, No. 23 Youzheng Road, \\ Nangang District, Harbin 150001, China. Email: hbwangwenbo_21@163.com.
}

Background: To investigate the role of microRNA-376b-3p (miR-376b-3p) and regulator of G protein signaling 1 (RGS1) in the proliferation, metastasis, and apoptosis of osteosarcoma.

Methods: Differentially expressed genes (DEGs) between tumor and normal tissues from GSE14359 and GSE33382 in the cancer genome atlas (TCGA) dataset were analyzed with GEO2R online. Similarly, differentially expressed miRNAs from GSE70367 were also analyzed with GEO2R. The interaction between the differentially expressed miRNAs and the shared distal metastasis-related DEGs from the two datasets were analyzed using miRWalk and Cytoscape. RGS1 and miR-376-3p were chosen to verify the prediction. RGS1 stably expressing and silencing cells were established based on the MG63 and U2OS cell lines. The targeting of RGS1 with miR-376b-3p was confirmed with Starbase prediction and luciferase reporter assay. Cell proliferation, metastasis, and apoptosis were characterized in vitro and in xenograft mice.

Results: A total of 10 up-regulated and 8 down-regulated DEGs were characterized as shared metastasisrelated DEGs for GSE14359 and GSE33382. Among these DEGs, RGS1 was targeted with miR-376b-3p, a predicted down-regulated miRNA in GSE70367. High expression of RGS1 predicted proliferation, invasion, metastases, and poor prognosis in osteosarcoma. Overexpression of RGS1 promoted proliferation, invasion, mobility, and stemness in MG63 and U2OS cells, while silencing of RGS1 had the opposite effect in both cell lines. High expression of RGS1 promoted tumor growth in xenograft nude mice. RGS1 was targeted with miR-376b-3p; the addition of miR-376b-3p down-regulated RGS1, and suppressed cell proliferation, invasion, and metastasis. Meanwhile, sponging of miR-376b-3p had the opposite effect. The suppressive effects of miR-376b-3p could be abolished with RGS1, as cell proliferation, stemness, metastasis, and invasion were all promoted with RGS1 co-transfection in both cell lines.

Conclusions: Our study indicated that RGS1 is a tumor-promoting gene in osteosarcoma, which could be inhibited with miR-376b-3p.

Keywords: Differently expressed genes (DEGs); miR-376b-3p; osteosarcoma; regulator of G protein signaling 1 (RGS1)

Submitted Aug 12, 2021. Accepted for publication Nov 16, 2021.

doi: $10.21037 / \mathrm{atm}-21-4949$

View this article at: https://dx.doi.org/10.21037/atm-21-4949 


\section{Introduction}

Osteosarcoma is a bone-forming tumor that mostly affects adolescents younger than 20 years of age (1). Despite its low incidence (approximately 5\% of all juvenile tumors), osteosarcoma accounts for the majority of pediatric cancer deaths. Sadly, treatment of osteosarcoma has essentially been unchanged for more than 30 years (2). For nonmetastatic osteosarcoma, a $70 \%$ overall survival rate is realizable. However, deadly lung metastasis develops easily in more than $80 \%$ of patients within 1 year of diagnosis (1). Exploring the pathology of osteosarcoma metastasis and malignancy is indispensable for better management of the disease.

Osteosarcoma is characterized by complex genomic instability and a high degree of genetic heterogeneity, and the resulting genetic alterations are related to copy number and genetic rearrangement (3). Genomic instability has been shown to cause changes in the cancer genome and microenvironment (4). Osteosarcoma exhibits epigenetic abnormalities, chromosomal rearrangement, point mutation, amplification, and loss of heterozygosity $(5,6)$. The genomic analysis revealed that the occurrence of osteosarcoma was mainly concentrated on early chromosomal changes. Previous studies detail the manner in which DNA copy number alterations are detailed, noting that chromosomal rearrangements can expand oncogenes and lead to reduced tumor suppressors, thereby accelerating tumor progression (7). Wu et al. found that osteosarcoma had a higher level of rearrangement than most other tumors (8). Chromosomal rearrangement and DNA copy number changes are the main driving forces for OS carcinogenesis.

With the development of microarray and multiple omics, bioinformatics has been a pivotal tool in discovering novel disease pathology and drug targets, including in osteosarcoma (9). On the other hand, limitations involving the quality of the dataset, the way of analysis, and the current understanding of the disease all make the analysis individualized and limited. In the current research, we explored the differentially expressed genes (DEGs) between tumor and normal tissues from GSE14359 and GSE33382 in the cancer genome atlas (TCGA) dataset, as well as differentially expressed microRNAs (miRNAs) from GSE70367. Here, we report for the first time that microRNA-376b-3p (miR-376b-3p) and regulator of $G$ protein signaling 1 (RGS1) are involved in the malignancy and metastasis of osteosarcoma. We present the following article in accordance with the ARRIVE reporting checklist (available at https://dx.doi.org/10.21037/atm-21-4949).

\section{Methods}

\section{GEO2R online analysis of DEGs}

The gene expression profiles of GSE70367, GSE14359, and GSE33382 were downloaded from TCGA datasets (https:// portal.gdc.cancer.gov/). For GEO2R analysis, Log2 I FoldChanges I $(\log 2 \mathrm{FC})>1$ and $\mathrm{P}<0.05$ was set as the screening criteria for DEGs. A Venn diagram was generated with DEGs online (http://bioinformatics.psb.ugent.be/webtools/ Venn/) in order to obtain shared intersection of different datasets. Finally, the intersection between differentially expressed miRNAs from GSE70367 and the shared DEGs for GSE14359 and GSE33382 were visualized using Cytoscape software (https://cytoscape.org/). The study was conducted in accordance with the Declaration of Helsinki (as revised in 2013).

\section{University of California, Santa Cruz (UCSC) Xena-based Gene Set Enrichment Analysis (GSEA)}

The TARET_OS dataset was downloaded from UCSC Xena; 14 genes that interact with specific miRNAs in GSE70367 and are involved in GSE14359 and GSE33382 were analyzed. RGS1 was chosen as it was possibly related to metastasis and malignancy. The specimen was divided into high and low RGS1 expression groups. GSEA analysis was carried out with java GSEA Desktop Application (http:// software.broadinstitute.org/gsea/downloads.jsp) using the default parameters to verify the relationship between RGS1 and the proliferation, metastasis, and invasion of osteosarcoma. Also, the specimens were divided into good and poor prognosis groups according to their outcomes, and the RGS1 levels between patients with good and poor prognoses were compared.

\section{Cell culture and transfection}

MG63 and U2OS cell lines (purchased from ATCC, Manassas, VA, USA) were cultured in Dulbecco's modified Eagle's medium containing high-glucose (DMEM-HG), supplemented with $10 \%$ fetal bovine serum (FBS) and $1 \%$ penicillin/streptomycin (all purchased from Gibco; Thermo Fisher Scientific, Inc., Waltham, MA, USA). The cells were cultured in a $37^{\circ} \mathrm{C}$ humidified incubator (Thermo Fisher Scientific, Inc., Waltham, MA, USA) with $5 \% \mathrm{CO}_{2}$. For transfection, a lentivirus-packaged RGS1 vector, short hairpin (sh) RNAs, miR-negative control (NC), miR376b-3p mimic, Sponge NC, and sponge miR-376b-3p 
were purchased from Hanyin Biotechology (Shanghai, China) (10). Cell transfection was applied according to the manufacturer's instructions. The levels of RGS1 protein in both RGS1 stably over-expressed and stably silenced cells were verified every 2 weeks with western blotting.

The sequence of the shRNAs and mimics were as follows: sh negative control (NC), 5'-CAGUACUUUUGU GUAGUACAAA-3'; shRGS1 \#1, 5'-GCATATCTAA GATCTATGATC-3'; shRGS1 \#2, 5'-GGGATGA AATCGTCCAAGTCC-3'; miR-NC, 5'-AAGAAUCAG GUUUUUCCAUGUU-3'; miR-376b-3p (mimic), 5 '-AUCAUAGAGGAAAAUCCAUGUU-3'; sponge NC, 5 '-AUCUUCGAAAUUCCUCUAUGUU-3'; and sponge miR-376b-3p, 5'-AACAUGGAUUUUCCUC UAUGAU-3'.

\section{Western blotting}

The treated cells were lysed with radio immunoprecipitation assay (RIPA) buffer (Beyotime, Nanjing, China) on ice. The supernatant of the lysis was collected after centrifugation for $15 \mathrm{~min}$ at $12,000 \mathrm{~g}$ and $4{ }^{\circ} \mathrm{C}$. The protein concentrations were tested using the Bradford protein assay kit (Beyotime, Shanghai, China). Each $30 \mu \mathrm{g}$ protein was added with loading buffer (Beyotime) and was heated to $100{ }^{\circ} \mathrm{C}$ for $10 \mathrm{~min}$. The samples were separated with $10 \%$ sodium dodecyl sulfate polyacrylamide gel electrophoresis (SDS-PAGE) gels (Beyotime, Shanghai, China), transferred to a 0.22 $\mu \mathrm{m}$ polyvinylidene difluoride (PVDF) membrane (Merck Millipore, Darmstadt, Germany), incubated with primary (anti-RGS1, ab154973, 1:500; anti- $\beta$-actin, ab8227, 1:3,000. Abcam, London, UK) and secondary (goat antirabbit immunoglobulin $\mathrm{G}$ (IgG), ab97051, 1:5,000, Abcam) antibodies, and visualized under a ChemiDoc XRS+ detector with chemiluminescent substrate ECL kit (Merck Millipore) according to the manufacturer's instructions. Finally, the visualized lanes were analyzed with Image J software (National Institutes of Health, USA).

\section{Cell viability}

After $24 \mathrm{~h}$ of transfection, approximately 5,000 cells were suspended in $1 \mathrm{~mL}$ of medium. $200 \mu \mathrm{L}$ medium was added into each well of a 96-well plate. The optical density of the plates was tested after incubation for 24, 48, 72, 96, and 120 h. Each well was added with $20 \mu \mathrm{L}$ of Cell Counting Kit (CCK)-8 $2 \mathrm{~h}$ prior to testing.

\section{Colony forming}

After $24 \mathrm{~h}$ of transfection, approximately 1,000 cells were suspended in $1 \mathrm{~mL}$ of medium (supplemented with $30 \%$ FBS), and added into a well of a six-well plate. The cells were cultured for 10 days and then stained with crystal violet. Cell colonies of more than 20 cells were counted under a microscope (Nikon, Japan).

\section{Wound bealing}

The transfected cells were cultured to reach a confluence of approximately $100 \%$. A wound was then straightly scratched with a sterile $200 \mu \mathrm{L}$ pipette tip, and $1 \%$ FBS medium was added. Wound healing was evaluated at 0 and $24 \mathrm{~h}$ with a light microscope (Nikon, Japan).

\section{Transwell assay}

For transwell assay, the upper chamber was precoated with $50 \mu \mathrm{L}$ Matrigel (Corning, NY, USA), and added with 30,000 transfected cells in $200 \mu \mathrm{L}$ FBS-free medium. The lower chamber was added with normal medium containing $10 \%$ FBS. The invasive cells were stained with crystal violet and observed under a microscope (Nikon, Japan).

\section{Detection of apoptosis}

To analyse the apoptosis of MG63 and U2OS cell lines, which were harvested and centrifuged for annexin V-FITC/ PI staining post infection $48 \mathrm{~h}$ with indicated plasmids or miRNAs. Briefly, transfected cells were incubated with $5 \mu \mathrm{L}$ annexin V-FITC and $10 \mu \mathrm{L}$ PI for $15 \mathrm{~min}$ in a dark room using an Annexin V-FITC Apoptosis Detection Kit (Beyotime, Shanghai, China). Finally, the apoptotic cells were analyzed using a flow cytometer (BD, USA). The apoptotic rate was defined as the percentage of early plus late apoptotic cells.

\section{Animal experiment}

In total, $10 \mathrm{BALB} / \mathrm{c}$ nude mice (male, weight 17-20 g, 6-weeks-old) were purchased from Charles River Inc. (Beijing, China). The mice were raised in a SPF house, with a $12 \mathrm{~h}$ day/night cycle, at $26.0{ }^{\circ} \mathrm{C}$ and $70 \%$ humidity. The empty vector or RGS1 stably transfected MG63 cells were seeded under the right flank skin at a density of $5 \times 10^{6}$ cells per nude mouse ( $\mathrm{n}=$ five mice per group). The 
tumor volume was recorded every 2 days. The mice were sacrificed 2 weeks later and the tumor volume and tumor weight were examined. This study was approved by the ethics committee of the animal center of Harbin Medical University (No. 2020-0026), and were performed according to the $3 \mathrm{R}$ principle and complied with the national standard for general requirements for animal experiments (GB/T 35823-2018) for the care and use of animals. A protocol was prepared before the study without registration.

\section{Luciferase reporter assay}

Starbase online (http://starbase.sysu.edu.cn/) was used to predict the targeting of RGS1 with miR-376b-3p. RGS1wild-type (WT, 5'-GACUAAAGUCUAUGAA-3'), RGS1mutant type (MUT, 5'-GACUAAAGAGAUACUA-3'), miR-NC, and miR-376b-3p were cloned inside a psi-check2 dual-luciferase reporter vector (Synbio Technologies, Suzhou, China), and then co-transfected into HEK-293T cells. After $48 \mathrm{~h}$ of incubation, the luciferase activity was measured according to the manufacturer's instructions (E292, Promega, USA).

\section{Quantitative reverse transcription-polymerase chain reaction ( $q R T-P C R)$}

Small RNA were extracted and separated using a miRNA isolation kit (OMEGA, China). The extracted small interfering (si) RNAs were then reversely transcribed using a Mir-X $\mathrm{X}^{\mathrm{TM}}$ miRNA First-Strand Synthesis kit (Takara, Japan). Next, a LightCycler 480 SYBR Green I Master kit (Roche, Basel, Switzerland) was used to run the cycles. The primers used were as follows: $\mathrm{miR}-376 \mathrm{~b}-3 \mathrm{p}$, forward 5'-GCCGAGATCATAGAGGAAAATC-3' and reverse 5'-CTCAACTGGTGTCGTGGA-3'; RGS1, forward 5 '-GCAGTGGTCTCAGTCTCTGGAAA-3' and reverse 5'-CAAAACATGTGGGAGTTGGTGTT-3'; glyceraldehyde-3-phosphate dehydrogenase (GAPDH), forward 5'-CAAGTTCAACGGCACAGTCAAGG-3' and reverse 5'-ACATACTCAGCACCAGCATCACC-3'; and U6, whose primers were provided within the Mir- $\mathrm{X}^{\mathrm{TM}}$ kit, was used as reference gene. The data were calculated using the Cq method.

\section{Statistical analysis}

All biological experiments were repeated at least three times. All quantitative data were expressed as mean \pm standard deviation. GraphPad Prism 5.0 software (GraphPad Software, San Diego, CA, USA) was used to analyze the data and plot the graphs. Differences were characterized with one-way analysis of variance (ANOVA) and Tukey's multiple comparisons.

\section{Results}

\section{DEGs in osteosarcoma and their interactions}

We characterized the DEGs in the tumor and normal tissues from GSE14359 and GSE33382 in TCGA dataset (Figure 1A). There were 195 shared up-regulated DEGs in the two datasets, of which 10 were metastasisrelated (Figure 1B). Also, there were 156 shared downregulated DEGs in the two datasets, of which eight were metastasis-related (Figure 1C). The fold change of the metastasis-related DEGs in the two datasets were analyzed (Figure 1D,1E), and HBB, APOC1, NES, and RGS1 were found to be the most DEGs. Differentially expressed miRNAs from GSE70367 were also analyzed (Figure 1F). The interaction between the differentially expressed miRNAs and the metastasis-related DEGs from GSE14359 and GSE33382 were analyzed (Figure 1G). HBB, CXCL10, IGSF6, and RGS1 were found to have broad interactions with these differentially expressed miRNAs.

\section{Correlation of RGS1 and malignant phenotype of osteosarcoma}

The malignant phenotype of osteosarcoma and the correlation with 14 metastasis-related and miRNAinteracting DEGs from GSE14359 and GSE33382 datasets were analyzed using the TARGET_OS dataset in UCSC Xena (data not shown). Among these DEGs, RGS1 was significantly related to the malignant phenotype of osteosarcoma, and was further investigated in this study. Specifically, RGS1 was related to the proliferation, migration, and invasion of osteosarcoma (Figure 2A-2C), and patients with worse prognoses had higher levels of RGS1 (Figure 2D). The results indicated that RGS1 is upregulated to promote osteosarcoma malignancy.

\section{Effect of RGS1 on the proliferation, migration, invasion and apoptosis of osteosarcoma cells}

RGS1 overexpression was verified with western blotting (Figure 3A). Compared with the Vector group, the RGS1 group showed a higher speed of proliferation (Figure 3B,3C), 


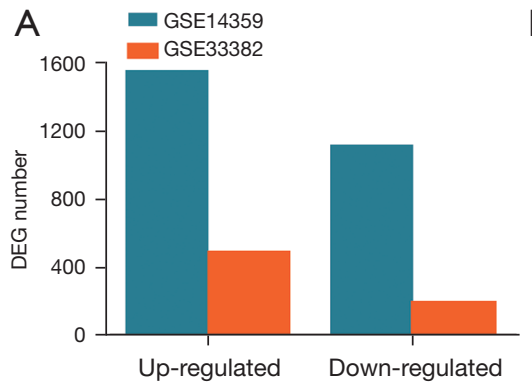

$\mathrm{D}$

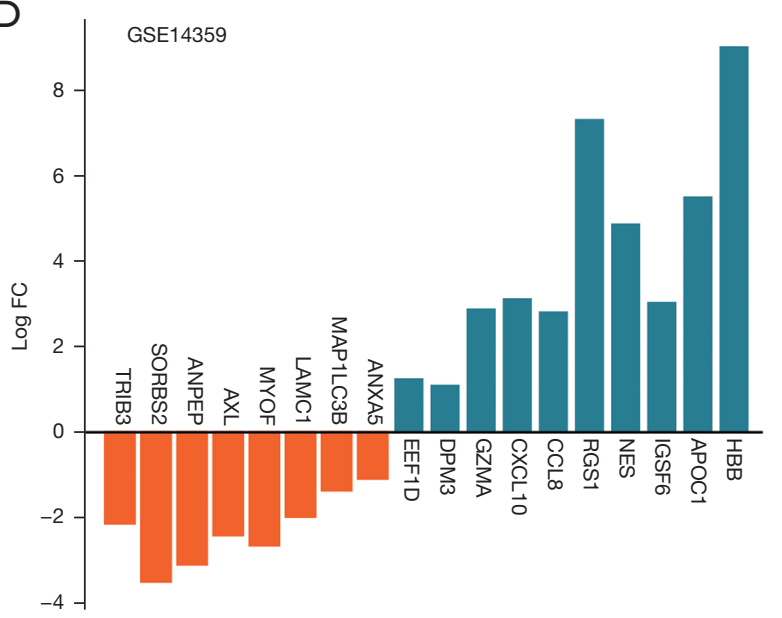

B

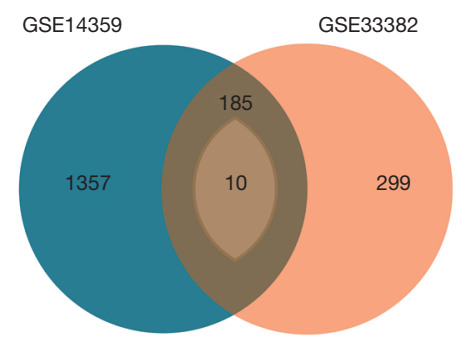

E
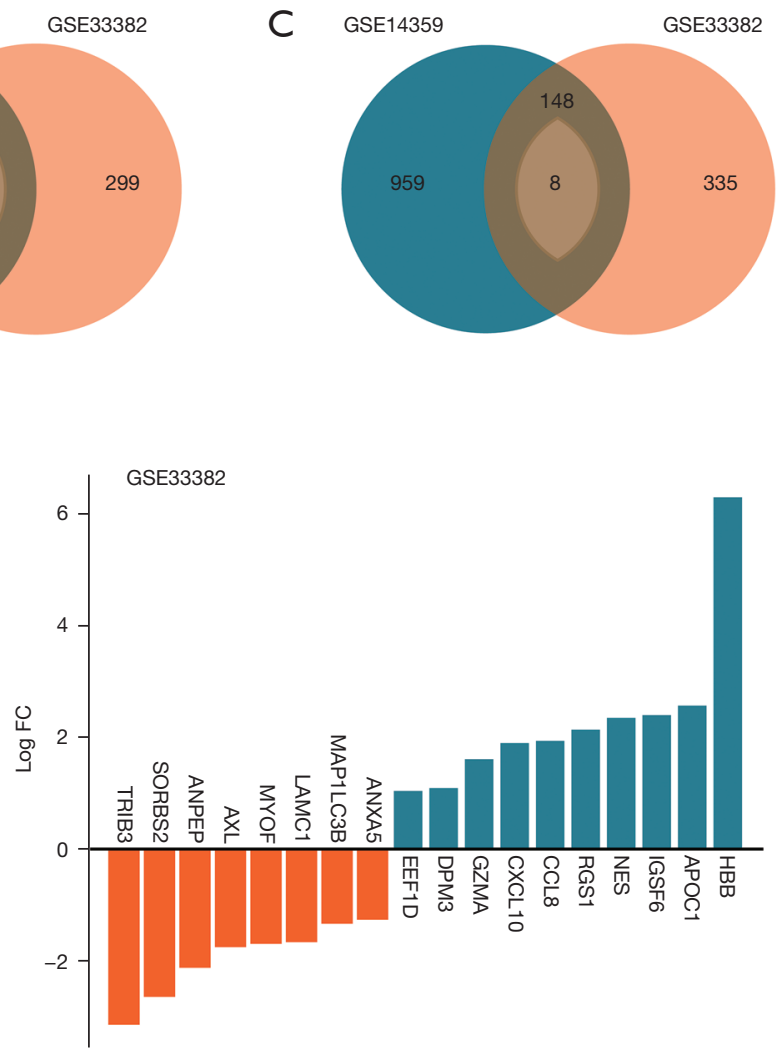

F

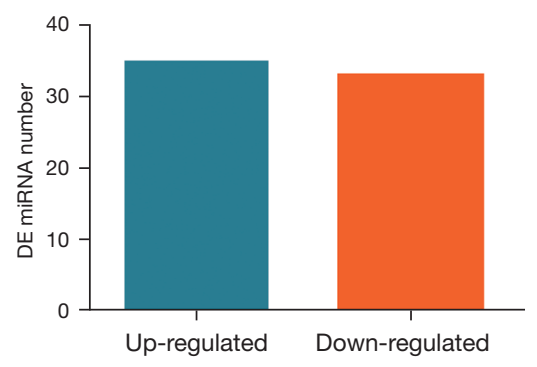

G

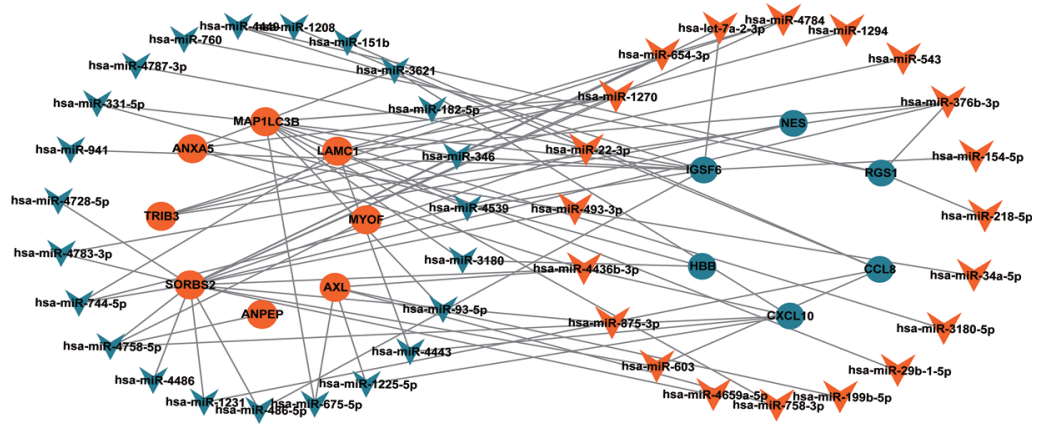

Figure 1 DEGs in osteosarcoma. (A) DEGs in tumor and normal tissues from GSE14359 and GSE33382 in TCGA dataset; (B) the shared up-regulated DEGs for GSE14359 and GSE33382, of which 10 were metastasis-related; (C) the shared down-regulated DEGs for GSE14359 and GSE33382, of which eight were metastasis-related; (D) the logFC of metastases-related DEGs in GSE14359; (E) the logFC of metastasis-related DEGs in GSE33382; (F) differentially expressed miRNAs in GSE70367; (G) the interaction between differentially expressed miRNAs and metastasis-related DEGs from GSE14359 and GSE33382. DEGs, differentially expressed genes; TCGA, the cancer genome atlas.

the colony forming rate was increased (Figure 3D), wound healing was expeditious (Figure $3 E, 3 F$ ), cell invasion was enhanced (Figure $3 G$ ), and apoptotic rate was reduced (Figure $3 H$ ) in both MG63 and U2OS osteosarcoma cells.

Also, RGS1 silencing was verified with western blotting (Figure 3I). Compared with shNC group, the shRGS1
1\# and shRGS1 2\# groups showed slower proliferation (Figure 37,3K), the colony forming rates were decreased (Figure $3 L$ ), wound healing was suppressed (Figure $3 M, 3 N$ ), cell invasion was inhibited (Figure 30 ), and apoptotic rate was increased (Figure $3 P$ ) in both MG63 and U2OS osteosarcoma cells. 

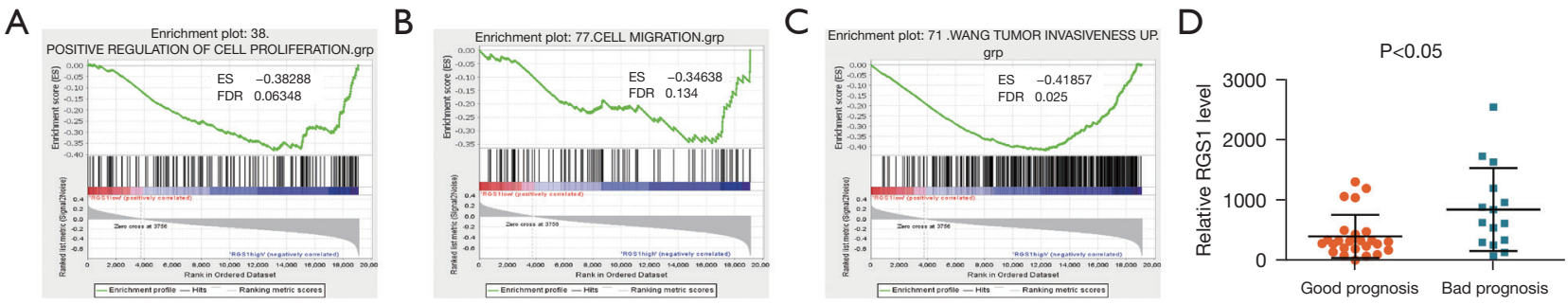

Figure 2 Relationship between RGS1 and the clinical malignant phenotype of osteosarcoma. (A) RGS1 enrichment between high and low proliferation specimens; (B) RGS1 enrichment between high and low migration specimens; (C) RGS1 enrichment between high and low invasion specimens; (D) RGS1 levels in specimens with good or poor prognosis. RGS1, regulator of G protein signaling 1.

The results indicated that RGS1 was up-regulated to promote the proliferation, migration, and invasion, and inhibit the apoptosis of osteosarcoma.

\section{Effect of RGS1 up-regulation on tumor growth in vivo}

MG63 cells transfected with the vector or RGS1 were subcutaneously injected in mice for 2 weeks. The tumors were subsequently removed from the mice and photographed (Figure 4A). The tumor volume was measured within 2 weeks; compared with the Vector group, the tumor volume in RGS1 group was increased (Figure $4 B$ ). The promotion of tumor growth with RGS1 was also supported by the tumor volume and weight measured on day 14 (Figure 4C,4D). The results suggested that RGS1 promoted tumor growth of osteosarcoma in vivo.

\section{RGS1 is targeted with miR-376b-3p}

The targeting of RGS1 with miR-376b-3p was also predicted in Starbase (Figure $5 A$ ). The luciferase reporter assay showed that HEK-293T cells co-transfected with RGS1-WT and miR-376b-3p had a significantly lower level of luciferase activity (Figure 5B). This further confirmed that RGS1 could be targeted with miR-376b-3p. Moreover, RGS1 messenger (m) RNA (Figure 5C) and protein (Figure 5D) levels in miR-376b-3p transfected MG63 and U2OS cells were lower than those in miR-NC transfected cells. These results indicated that miR-376b-3p targeting of RGS1 reduced its expression in MG63 and U2OS osteosarcoma cells.

\section{Effect of miR-376b-3p on the proliferation, migration, invasion and apoptosis of osteosarcoma cells}

MiR-376b-3p overexpression was verified by western blotting (Figure 6A). Compared with miR-NC group, the miR-376b-3p group exhibited a lower speed of proliferation (Figure $6 B, 6 C$ ), the colony forming rate was decreased (Figure $6 D$ ), wound healing was inhibited (Figure $6 E, 6 F$ ), cell invasion was reduced (Figure $6 G$ ), and apoptotic rate was increased (Figure 6H) both in MG63 and U2OS osteosarcoma cells.

MiR-376b-3p sponging was also verified by western blotting (Figure 6I). Compared with sponge NC group, the sponge miR-376b-3p group showed a lower speed of proliferation (Figure $67,6 \mathrm{~K}$ ), the colony forming rate was decreased (Figure $6 L$ ), wound healing was faster (Figure 6M,6N), cell invasion was enhanced (Figure 6O), and apoptotic rate was reduced (Figure 6P) both in MG63 and U2OS osteosarcoma cells.

These results indicated that RGS1 could be targeted by miR-376b-3p to inhibit the proliferation, migration, and invasion, and induce the apoptosis of osteosarcoma.

\section{Effect of miR-376b-3p and RGS1 co-transfection on the proliferation, migration, invasion and apoptosis of osteosarcoma cells}

The inhibitory effect of RGS1 on miR-376b-3p was verified. Compared with miR-NC, RGS1 was decreased in the miR376b-3p group; however, compared with the miR-376b$3 p$ group, RGS1 was increased in the miR-376b-3p and RGS1 co-transfection group in both MG63 and U2OS cells (Figure $7 A$ ). Similarly, compared with miR-NC, the cell viability (Figure $7 B, 7 C$ ), relative colony number (Figure $7 D$ ), wound healing (Figure $7 E, 7 F$ ), and invasion (Figure $7 G$ ) were all inhibited in the miR-376b-3p group. In addition, compared with miR-NC, the apoptotic rate (Figure $7 H$ ) was increased. However, all of these were reverted in miR-376b$3 p$ and RGS1 co-transfection group, which indicated that the inhibitory effect of miR-376b-3p was reversed by RGS1. 


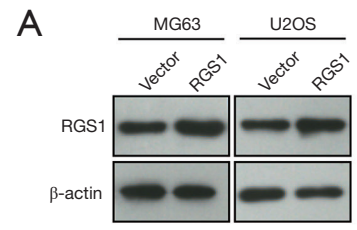

D
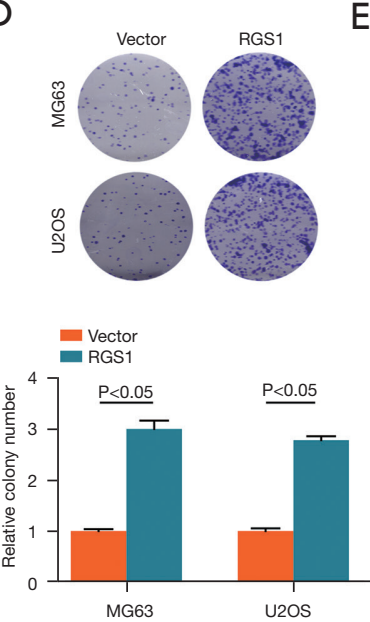

G
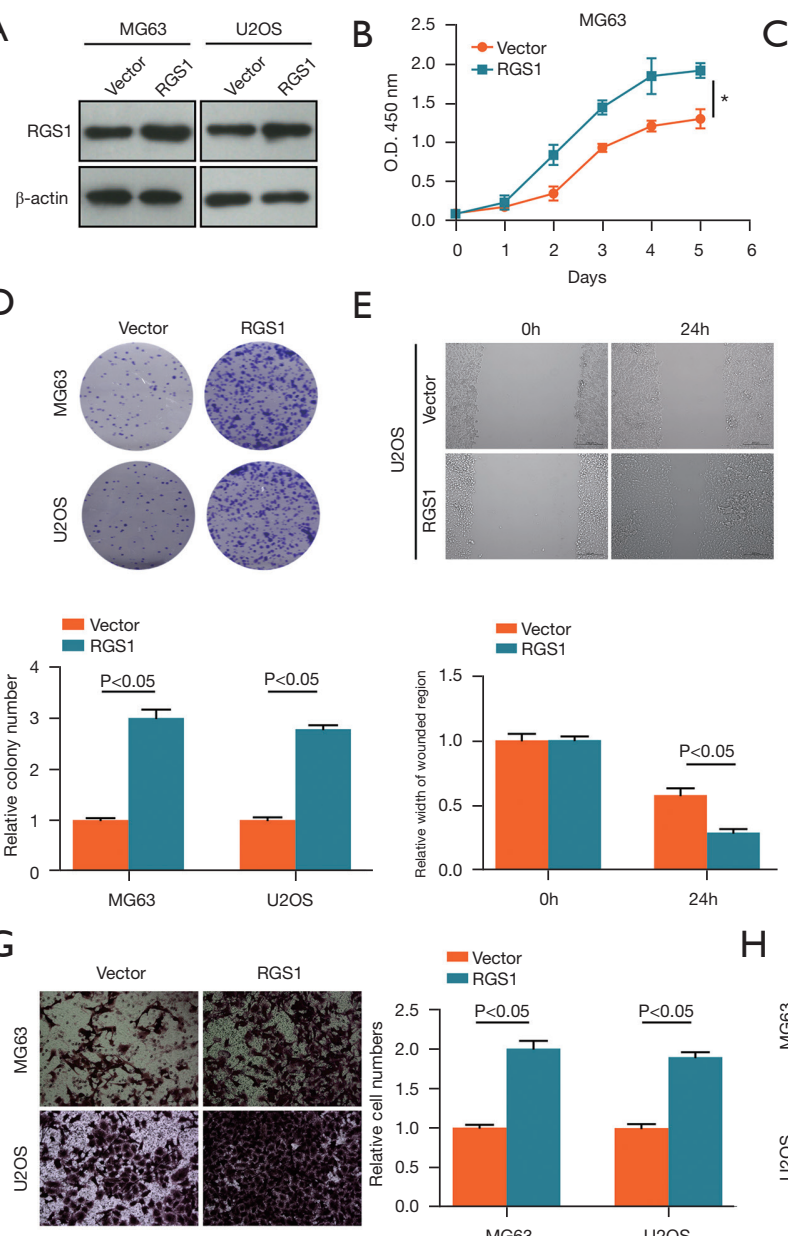

E
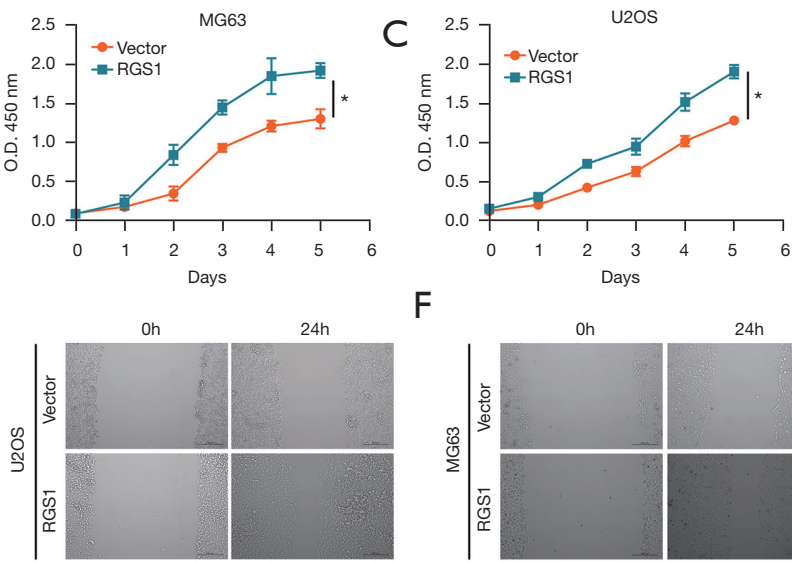

$\mathrm{F}$
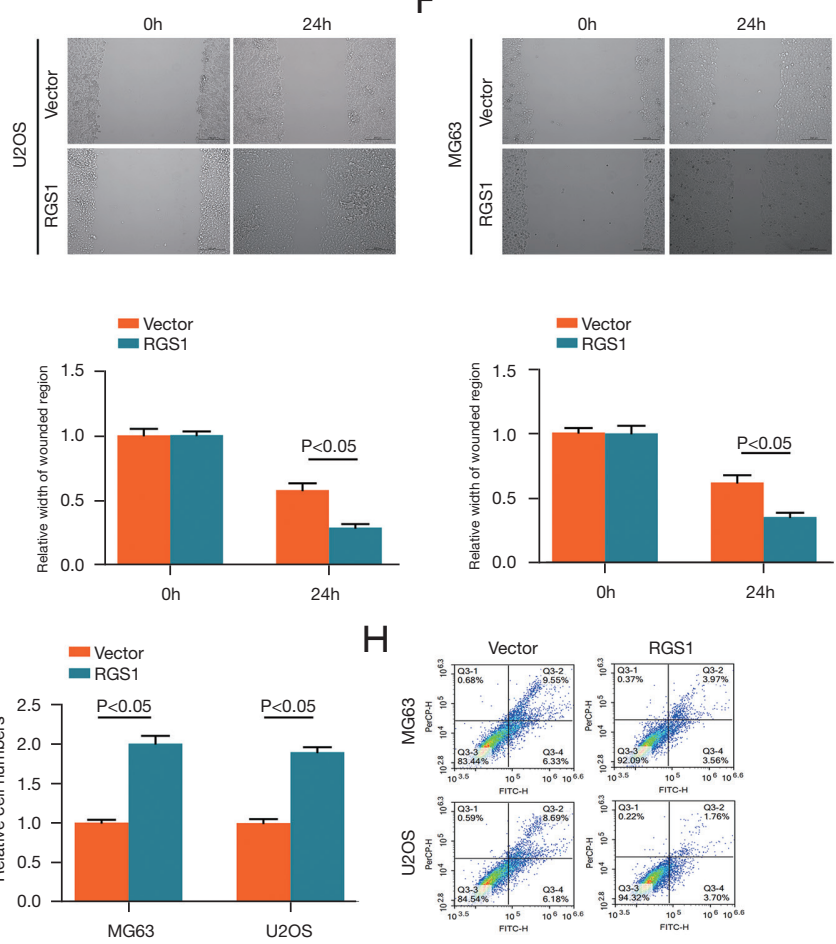

$\mathrm{H}$
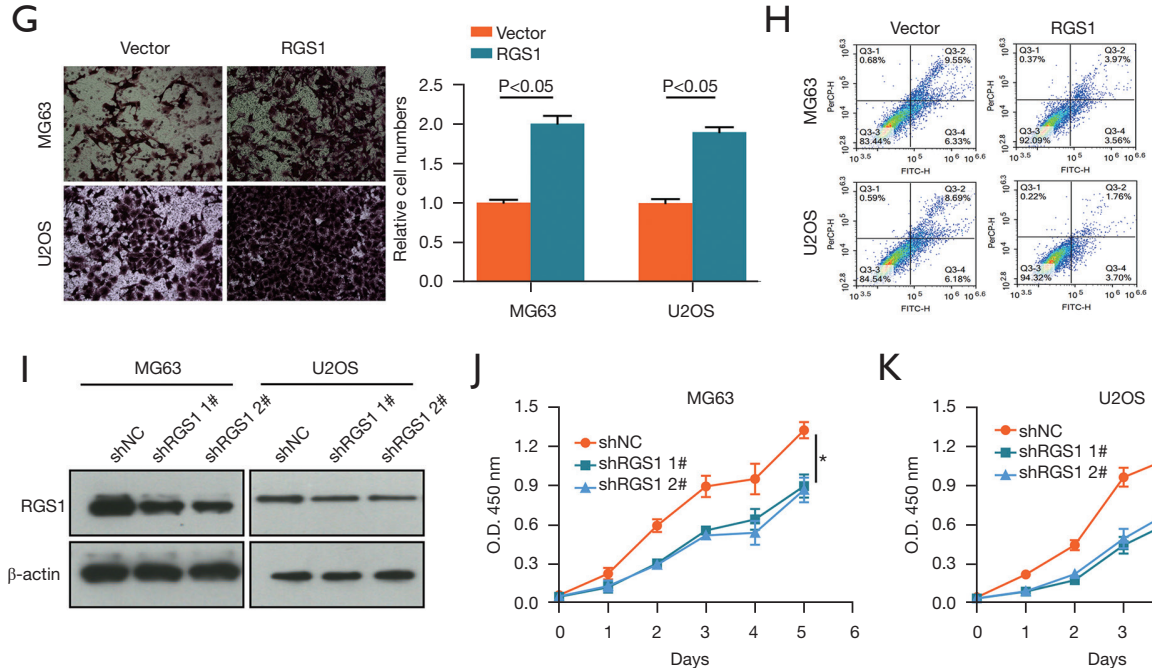

K

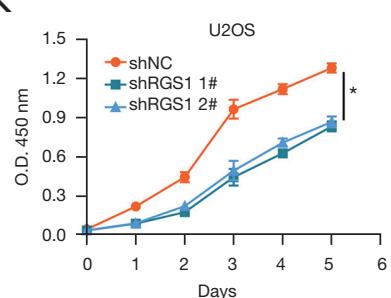


$\mathrm{L}$

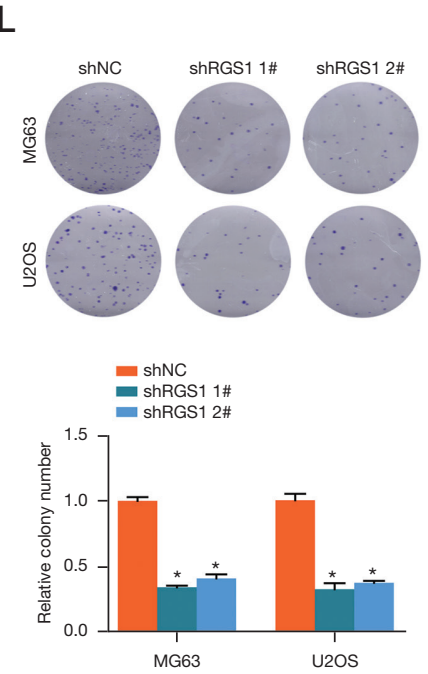

M
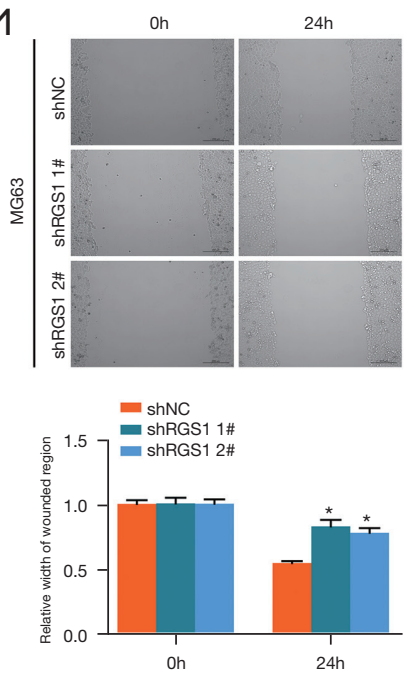
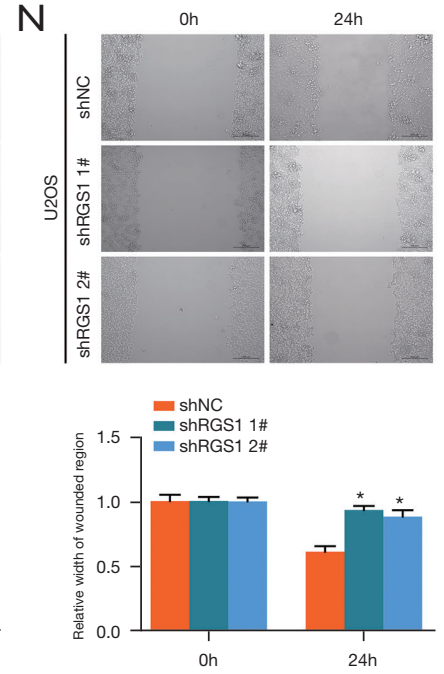
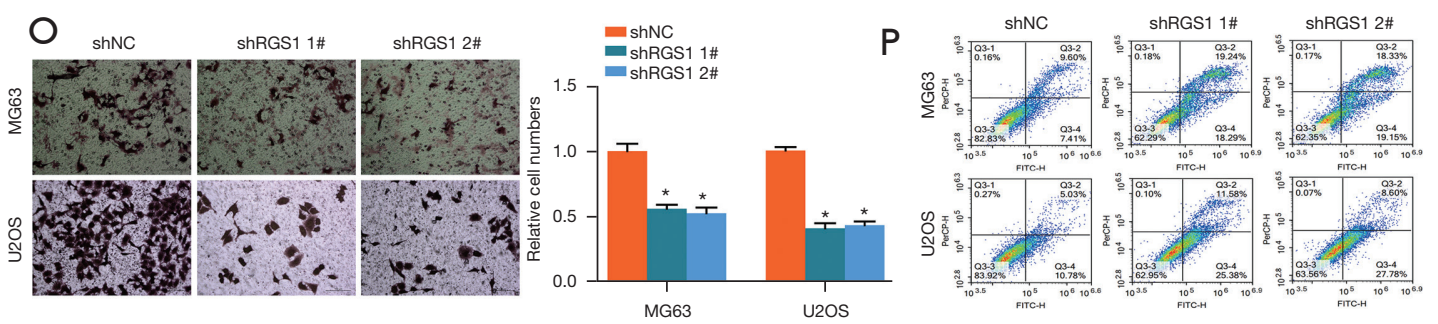

Figure 3 Effect of RGS1 on the proliferation, migration, invasion, and apoptosis of osteosarcoma cells. (A) Validation of RGS1 high expression in MG63 and U2OS cells; (B) MG63 cell viability; (C) U2OS cell viability; (D) colony forming of MG63 and U2OS cells, which were stained with crystal violet; (E) wound healing of MG63 cells, images were magnified at ×100; (F) wound healing of U2OS cells, images were magnified at $\times 100 ;(\mathrm{G})$ invasive ability of MG63 and U2OS cells, which were stained with crystal violet, and images were magnified at ×200; (H) apoptotic rate of MG63 and U2OS cells; (I) validation of RGS1 silencing in MG63 and U2OS cells; (J) MG63 cell viability; (K) U2OS cell viability; (L) colony forming of MG63 and U2OS cells, which were stained with crystal violet; (M) wound healing of MG63 cells, images were magnified at $\times 100 ;(N)$ wound healing of $\mathrm{U} 2 \mathrm{OS}$ cells, images were magnified at $\times 100 ;(\mathrm{O})$ invasive ability of MG63 and U2OS cells, which were stained with crystal violet, and images were magnified at $\times 200$. (P) apoptotic rate of MG63 and U2OS cells. $\mathrm{N}=6,{ }^{*} \mathrm{P}<0.05$ versus Vector by $t$-test or one-way ANOVA. RGS1, regulator of G protein signaling 1; ANOVA, analysis of variance.
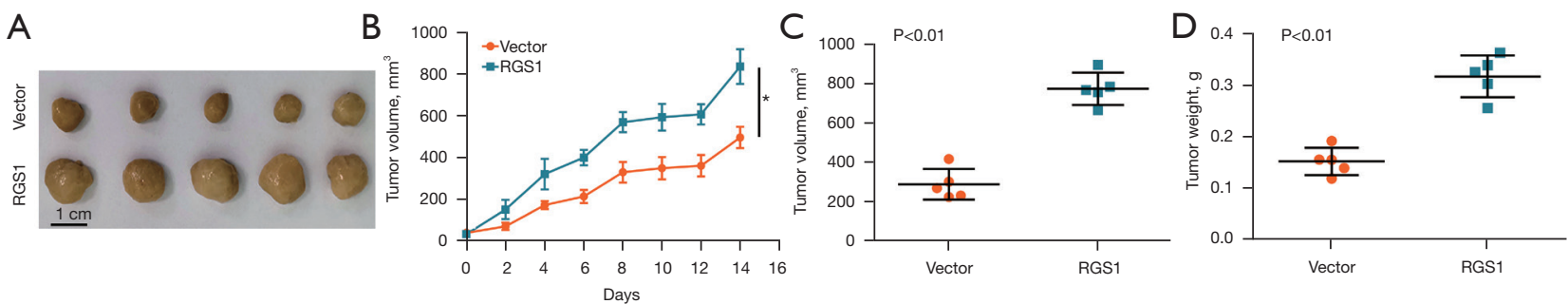

Figure 4 Effect of RGS1 overexpression on the tumor growth of MG63 cells xenograft mice. (A) The tumors formed in 2 weeks; (B) tumor size was measured within 2 weeks; (C) tumor volume at day 14; (D) tumor weight at day 14 . $\mathrm{N}=5$, ${ }^{*} \mathrm{P}<0.05$ versus Vector by $t$-test or one-way ANOVA. RGS1, regulator of G protein signaling 1; ANOVA, analysis of variance. 

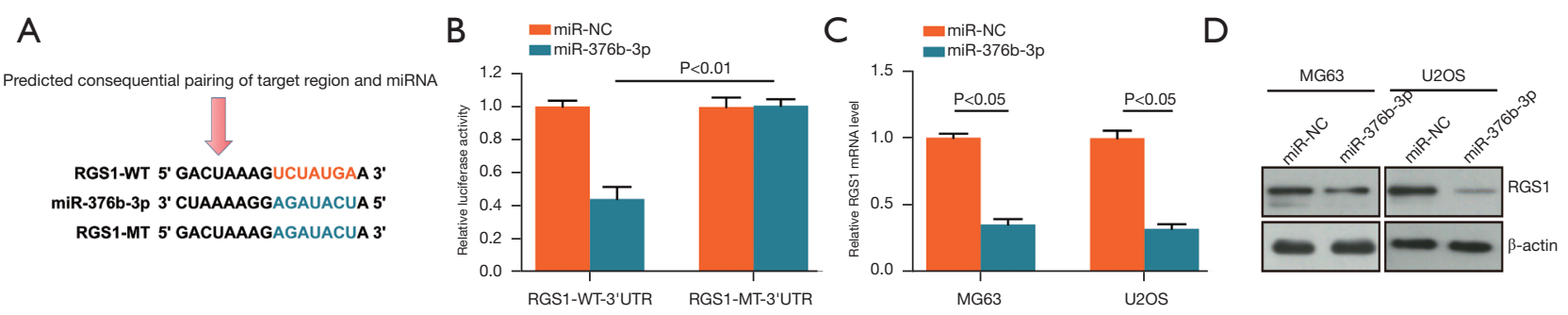

Figure 5 The targeting of RGS1 with miR-376b-3p. (A) Starbase predicted the interaction of RGS1 with miR-376b-3p and the mutant RGS1; (B) the targeting of RGS1 with miR-376b-3p was certified by luciferase reporter assay; (C) RGS1 mRNA expression in miR-376b3p-transfected MG63 and U2OS cells; (D) levels of RGS1 in miR-376b-3p-transfected MG63 and U2OS cells. N=6, P by t-test was indicated with line segments between different groups. RGS1, regulator of G protein signaling 1 .

\section{Discussion}

Osteosarcoma is the most common subtype of bone cancer in children and young adults (1). The 5 -year survival rate is below $30 \%$ for metastatic or recurrent patients, and recent progression in tumor treatment has proven ineffective in osteosarcoma (2). Gene alterations in osteosarcoma are mainly copy number changes, with few tumorigenic point mutations, which is not the prevalent situation in most tumors (11). It is important to explore the pathology of osteosarcoma with respect to its genomic landscape, so that effective drug targets can be characterized. In this study, the mRNA and miRNA expression in osteosarcoma was analyzed with GSE14359, GSE33382, and GSE70367. Metastasis-related DEGs were analyzed, of which RGS1 was further investigated as it has a high $\operatorname{LogFC}$. It has been found to be associated with clinical malignant phenotypes of tumors, but has rarely been studied in osteosarcomas. RGS1 is currently characterized as a proinflammatory regulator that is correlated with the poor differentiation and prognosis of several tumors (12-16). RGS1 is up-regulated to promote melanoma progression through phosphorylation of protein kinase B (AKT) and extracellular regulated protein kinases (ERK) (10); RGS1 can also regulate the immunophenotype of alveolar macrophage subpopulations, including inflammatory response, injury repair, and fibrosis phenotypes, via intracellular calcium $(\mathrm{Ca})^{2+}$ regulation (17). During vitiligo development, RGS1 overexpression is related to cluster of differentiation $(\mathrm{CD}) 4^{+} \mathrm{T}$ cell abundance (18). High expression of RGS1 is closely related to the progression and prognosis of several tumors, including gastric cancer (19) and hepatocellular carcinoma (13). Chromosomal rearrangement occurs in the development of lymphoid cells (20). Similarly, chromosomal rearrangement in osteosarcoma promotes tumor malignancy (8), which could possibly be regulated with the immunophenotyperegulating gene, RGS1. Furthermore, CXCL10, an upregulated metastases-related DEG predicted in this study, is also an immunophenotype-regulating gene. We predicted that CCL8, as an up-regulated metastasis-related DEG (and CCL18, a similar protein) is also an immunophenotyperegulating gene (18). There may be some similarities between immunophenotype-regulating and osteosarcoma malignant phenotype development. In this study, we found that RGS1 is up-regulated in patients with poor prognosis, and is related to the proliferation, migration, and invasion of osteosarcoma. Over-expression of RGS1 promoted the proliferation, migration, and invasion of MG63 and U2OS osteosarcoma cells, while silencing of RGS1 inhibited the proliferation, migration, and invasion of MG63 and U2OS osteosarcoma cells. Furthermore, the tumor-promoting effect of RGS1 was verified with RGS1 overexpressing cells in xenograft mice. RGS1-transfected NG63 cells grew faster within 2 weeks in mice. Our results supported RGS1 as a tumor-promoting gene in osteosarcoma.

MiR-376b-3p is a tumor-suppressive gene that is down-regulated in multiple tumors (21-23). For example, miR-376b-3p has been shown to be more significantly differentially expressed in the tumors of patients with primary resistance of renal cell carcinoma patients (24). In the serum exosomes of patients with malignant glioma, miR-376b-3p is markedly reduced; however, the upregulation of miR-376b-3p inhibits the proliferation, invasion, and formation of vasculogenic mimicry in U87 cells (25). In the present study, we showed that miR-376b$3 p$ targeted RGS1 to reduce its expression. MiR-376b-3p up-regulation inhibited the proliferation, migration, and invasion of MG63 and U2OS osteosarcoma cells. These tumor-suppressive effects of miR-376b-3p were abolished 


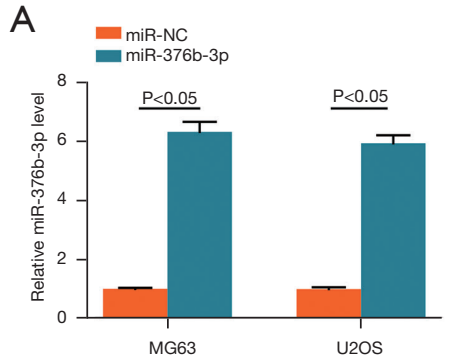

B

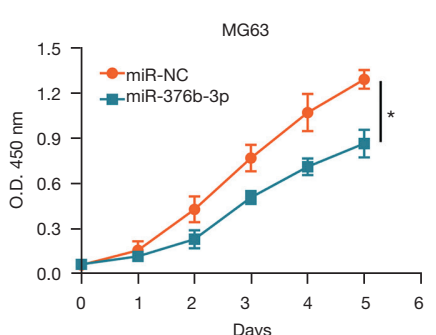

E
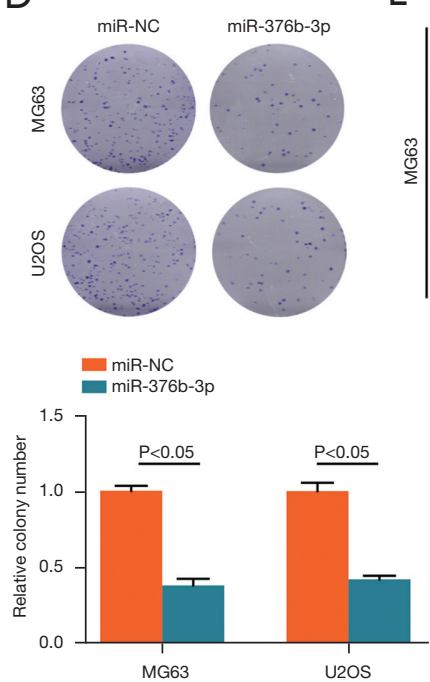

G

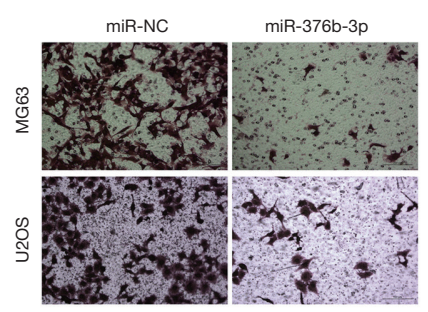

I $\quad$ Sponge NC J

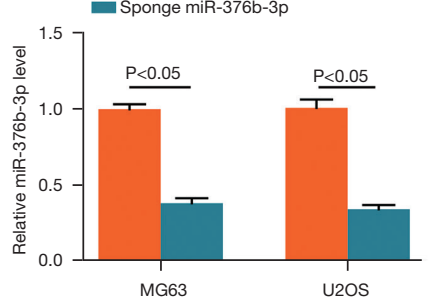

C

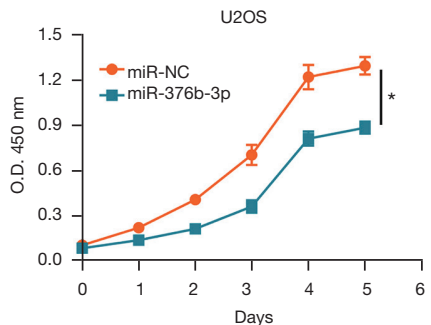

F
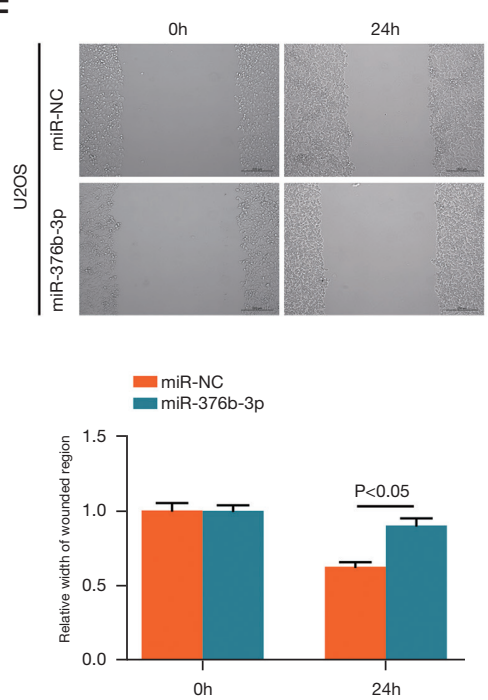

$\mathrm{H}$

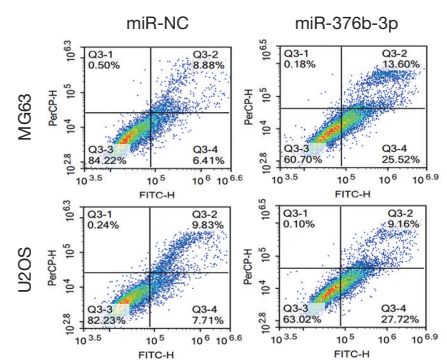

K

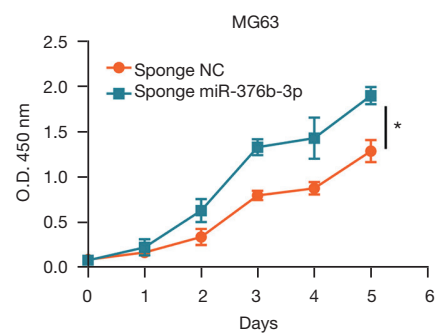

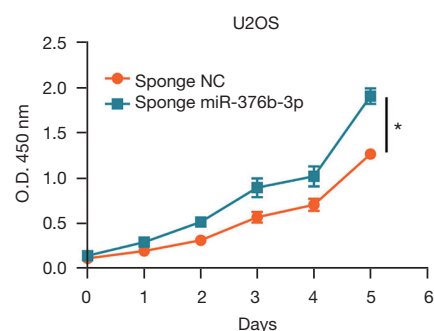


$\mathrm{L}$
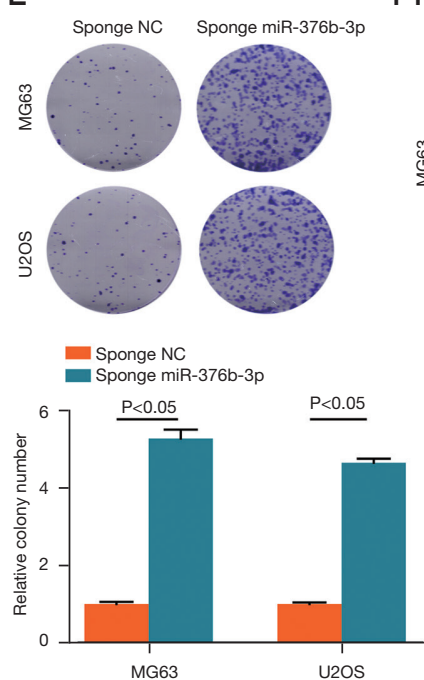

O

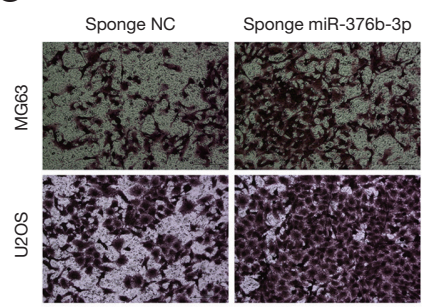

M

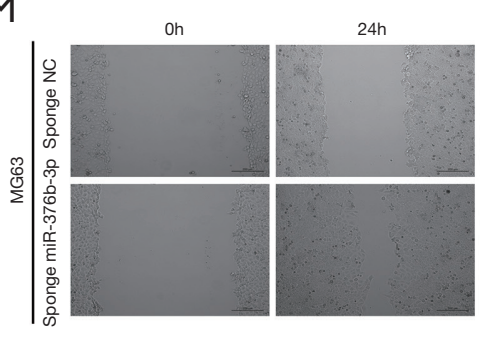

Sponge NC

Sponge miR-376b-3p

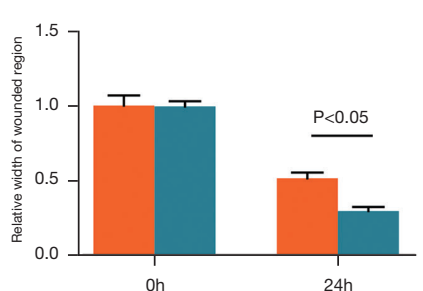

$24 \mathrm{~h}$

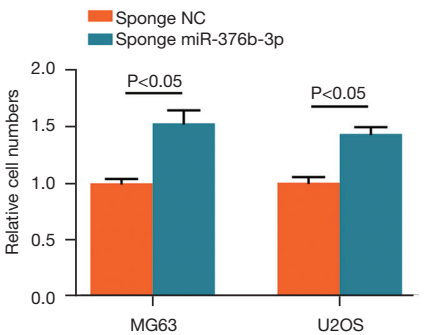

$\mathrm{N}$

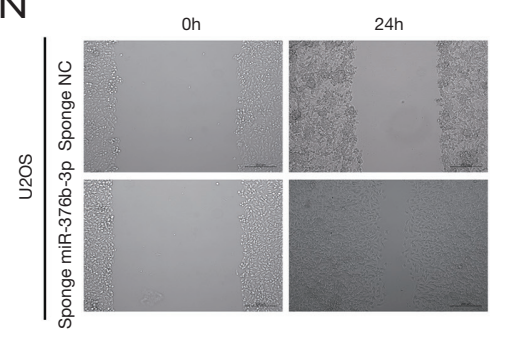

Sponge NC Sponge miR-376b-3p

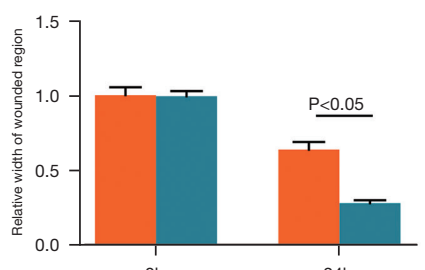

Oh

$P$

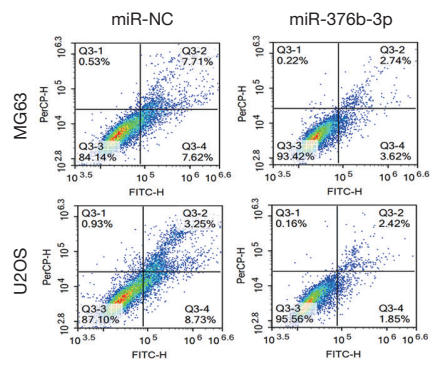

Figure 6 Effect of miR-376b-3p on the proliferation, migration, invasion, and apoptosis of MG63 and U2OS cells. (A) Validation of miR376b-3p transfection; (B) viability of MG63 cells within 5 days; (C) viability of U2OS cells within 5 days; (D) colony forming of MG63 and U2OS cells, which were stained with crystal violet; (E) wound healing of MG63 cells, images were magnified at $\times 100$; (F) wound healing of U2OS cells, images were magnified at $\times 100$; (G) invasive ability of MG63 and U2OS cells, which were stained with crystal violet, and images were magnified at 200×; (H) apoptotic rate of MG63 and U2OS cells; (I) validation of miR-376b-3p sponging; (J) MG63 cell viability; (K) U2OS cell viability; (L) colony forming of MG63 and U2OS cells, which were stained with crystal violet; (M) wound healing of MG63 cells, images were magnified at $\times 100$; $(\mathrm{N})$ wound healing of U2OS cells, images were magnified at $\times 100 ;(\mathrm{O})$ invasive ability of MG63 and U2OS cells, which were stained with crystal violet, and images were magnified at $\times 200$; (P) apoptotic rate of MG63 and U2OS cells. $\mathrm{N}=6,{ }^{*} \mathrm{P}<0.05$ versus Vector by $t$-test or one-way ANOVA. ANOVA, analysis of variance.

by RGS1 co-transfection. These results indicated that miR$376 \mathrm{~b}-3 \mathrm{p}$ could target RGS1 to inhibit the proliferation, migration, and invasion of osteosarcoma cells.

Two miRNAs, miR-376b-3p and miR-218-5p, were predicted to be down-regulated miRNAs that possibly targeted RGS1. MiR-218-5p was verified as a tumor suppressor in osteosarcoma, while miR-218-5p could possibly be sponged by several long surviving RNAs, including circ_0028171 (26), circSAMD4A (27), and lncRNA NR2F1 (28). Currently, there are no reports on miR-376b-3p in osteosarcoma. Whether miR-376b-3p could effectively exert its tumor-suppressive effects in vivo needs to be further investigated. This study for the first time confirmed the inhibitory effect of miR-376b-3p in osteosarcoma cells, providing a new idea for osteosarcoma targeted therapy, which was conducive to the further treatment and prognosis of patients with osteosarcoma.

In conclusion, our results suggested that RGS1 is upregulated in osteosarcoma to he promote proliferation, migration, and invasion, which could be inhibited by miR376b-3p. The role of RGS1, especially in phenotype regulation, should be further explored in detail to determine its potential value in the diagnosis and treatment of osteosarcoma. 

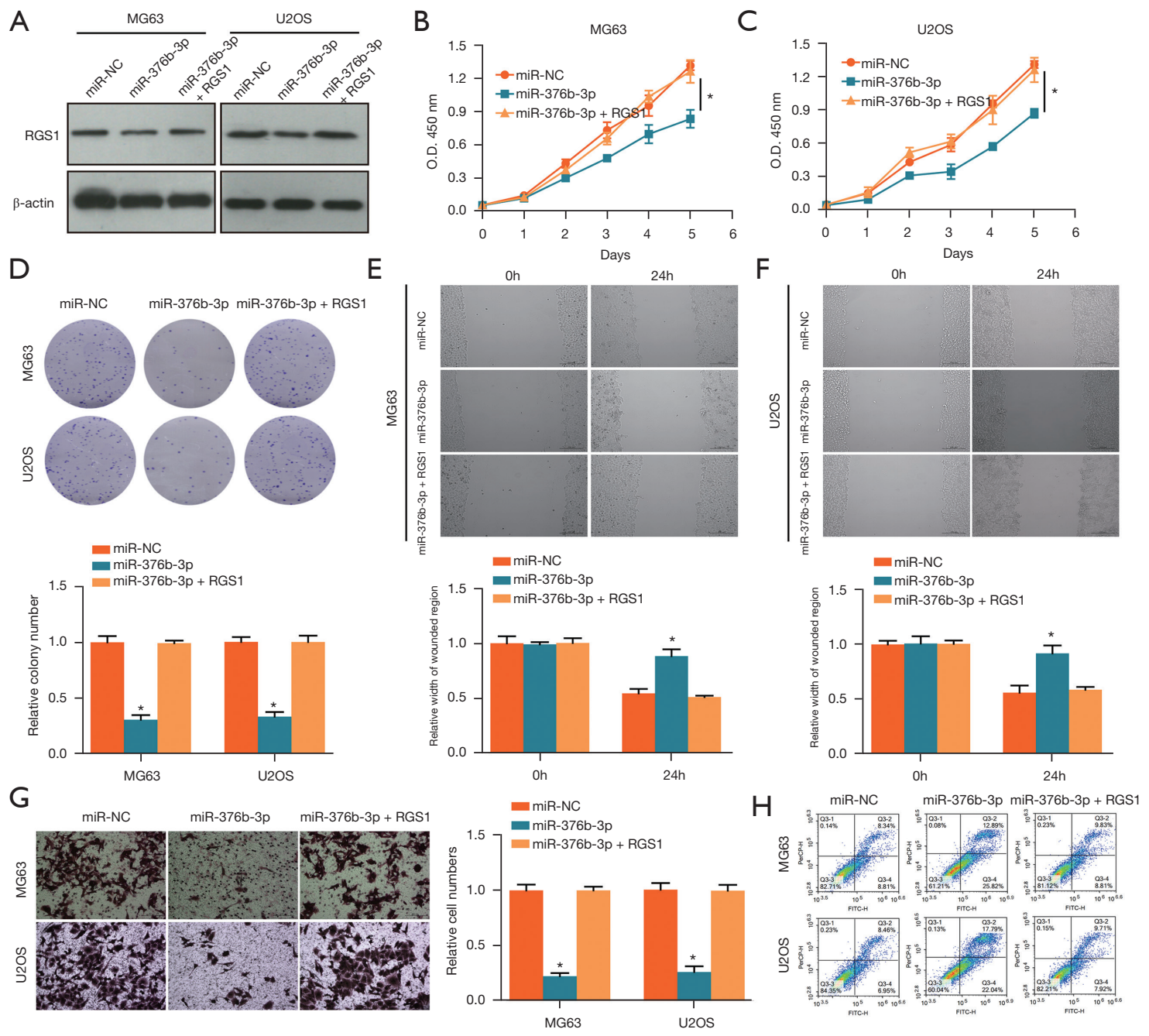

Figure 7 Effect of miR-376b-3p and RGS1 co-transfection on the proliferation, migration, invasion, and apoptosis of osteosarcoma cells. (A) Protein expression of RGS1; (B) viability of MG63 cells within 5 days; (C) viability of U2OS cells within 5 days; (D) colony forming of MG63 and U2OS cells, which were stained with crystal violet; (E) wound healing of MG63 cells, images were magnified at $\times 100$; (F) wound healing of U2OS cells, images were magnified at 100x; (G) invasive ability of MG63 and U2OS cells, which were stained with crystal violet, and images were magnified at 200x; (H) apoptotic rate of MG63 and U2OS cells. $\mathrm{N}=6,{ }^{*} \mathrm{P}<0.05$ versus Vector by $t$-test or one-way ANOVA. RGS1, G protein signaling 1; ANOVA, analysis of variance.

\section{Acknowledgments}

Funding: None.

\section{Footnote}

Reporting Checklist: The authors have completed the ARRIVE reporting checklist. Available at https://dx.doi. org/10.21037/atm-21-4949
Data Sharing Statement: Available at https://dx.doi. org/10.21037/atm-21-4949

Conflicts of Interest: All authors have completed the ICMJE uniform disclosure form (available at https://dx.doi. org/10.21037/atm-21-4949). The authors have no conflicts of interest to declare.

Ethical Statement: The authors are accountable for all 
aspects of the work in ensuring that questions related to the accuracy or integrity of any part of the work are appropriately investigated and resolved. The study was conducted in accordance with the Declaration of Helsinki (as revised in 2013). This study was approved by the ethics committee of the animal center of Harbin Medical University (No. 2020-0026), and were performed according to the $3 \mathrm{R}$ principle and complied with the national standard for general requirements for animal experiments (GB/T 35823-2018) for the care and use of animals.

Open Access Statement: This is an Open Access article distributed in accordance with the Creative Commons Attribution-NonCommercial-NoDerivs 4.0 International License (CC BY-NC-ND 4.0), which permits the noncommercial replication and distribution of the article with the strict proviso that no changes or edits are made and the original work is properly cited (including links to both the formal publication through the relevant DOI and the license). See: https://creativecommons.org/licenses/by-nc-nd/4.0/.

\section{References}

1. Lilienthal I, Herold N. Targeting molecular mechanisms underlying treatment efficacy and resistance in osteosarcoma: a review of current and future strategies. Int J Mol Sci 2020;21:6885.

2. Xie X, Zeng Z, Tu J, et al. Regional lymph node involvement is associated with poorer survivorship in patients with upper extremity osteosarcoma than with lower extremity os-teosarcoma: a SEER analysis. Transl Cancer Res 2020;9:7022-33.

3. Behjati S, Tarpey PS, Haase K, et al. Recurrent mutation of IGF signaling genes and distinct patterns of genomic rearrangement in osteosarcoma. Nat Commun 2017;8:15936.

4. Chen X, Bahrami A, Pappo A, et al. Recurrent somatic structural variations contribute to tumorigenesis in pediatric osteosarcoma. Cell Rep 2014;7:104-12.

5. Gröbner SN, Worst BC, Weischenfeldt J, et al. The landscape of genomic alterations across childhood cancers. Nature 2018;555:321-7.

6. Tsiambas E, Fotiades PP, Sioka C, et al. Novel molecular and metabolic aspects in osteosarcoma. J BUON 2017;22:1595-8.

7. Saraf AJ, Fenger JM, Roberts RD. Osteosarcoma: accelerating progress makes for a hopeful future. Front Oncol 2018;8:4.
8. Wu CC, Livingston JA. Genomics and the immune landscape of osteosarcoma. Adv Exp Med Biol. 2020;1258:21-36.

9. Zhou X, Fan $Y, Y e W$, et al. Identification of the novel target genes for osteosarcoma therapy based on comprehensive bioinformatic analysis. DNA Cell Biol 2020;39:1172-80.

10. Sun MY, Wang Y, Zhu J, et al. Critical role for nonGAP function of Gas in RGS1-mediated promotion of melanoma progression through AKT and ERK phosphorylation. Oncol Rep 2018;39:2673-80.

11. Ciriello G, Miller ML, Aksoy BA, et al. Emerging landscape of oncogenic signatures across human cancers. Nat Genet 2013;45:1127-33.

12. Zhu T, Lou Q, Shi Z, et al. Identification of key miRNAgene pairs in gastric cancer through integrated analysis of mRNA and miRNA microarray. Am J Transl Res 2021;13:253-69.

13. Ranjpour M, Wajid S, Jain SK. Elevated expression of sepiapterin reductase, regulator of $\mathrm{G}$ protein signaling 1 , hypothetical protein CXorf58 homolog, and zinc finger and BTB domain-containing protein 21 isoform $\mathrm{X} 2$ is associated with progression of hepatocellular carcinoma. Protoplasma 2021;258:1133-43.

14. Li Y, Liu M, Yang S, et al. RGS12 is a novel tumor suppressor in osteosarcoma that inhibits YAP-TEAD1Ezrin signaling. Oncogene 2021;40:2553-66.

15. Hu Y, Zheng M, Wang S, et al. Identification of a fivegene signature of the RGS gene family with prognostic value in ovarian cancer. Genomics 2021;113:2134-44.

16. Wang Y, Liu G, Ren L, et al. Long non-coding RNA TUG1 recruits miR-29c-3p from its target gene RGS1 to promote proliferation and metastasis of melanoma cells. Int J Oncol 2019;54:1317-26.

17. Feng Z, Zhou J, Liu Y, et al. Epithelium- and endotheliumderived exosomes regulate the alveolar macrophages by targeting RGS1 mediated calcium signaling-dependent immune response. Cell Death Differ 2021;28:2238-56.

18. Zhang J, Yu S, Hu W, et al. Comprehensive analysis of cell population dynamics and related core genes during vitiligo development. Front Genet 2021;12:627092.

19. Li S, Yang H, Li S, et al. High expression of regulator of G-protein signalling 1 is associated with the poor differentiation and prognosis of gastric cancer. Oncol Lett 2021;21:322.

20. Ji Y, Zhang J, Lee AI, et al. A multistep mechanism for the activation of rearrangement in the immune system. Proc Natl Acad Sci U S A 2003;100:7557-62. 


\section{Page 14 of 14}

21. Zhu D, Xiao Z, Wang Z, et al. MEG3/MIR-376B-3P/ HMGA2 axis is involved in pituitary tumor invasiveness. J Neurosurg 2020;3;1-13.

22. Li P, Cai JX, Han F, et al. Expression and significance of miR-654-5p and miR-376b-3p in patients with colon cancer. World J Gastrointest Oncol 2020;12:492-502.

23. Chen LM, Niu YD, Xiao M, et al. LncRNA NEAT1 regulated cell proliferation, invasion, migration and apoptosis by targeting has-miR-376b-3p/SULF1 axis in non-small cell lung cancer. Eur Rev Med Pharmacol Sci 2020;24:4810-21.

24. Kovacova J, Juracek J, Poprach A, et al. MiR-376b$3 \mathrm{p}$ is associated with long-term response to sunitinib in metastatic renal cell carcinoma patients. Cancer Genomics Proteomics 2019;16:353-9.

25. Jiang J, Wang S, Meng QH, et al. Study on the expression

\section{Zhang et al. miR-376b-3p targets RGS1 to inhibit osteosarcoma}

of non-coding microRNA-376b-3p in serum exosomes of patients with malignant glioma and the mechanism of antiangiogenesis. Zhonghua Yi Xue Za Zhi 2020;100:1634-9.

26. Pan F, Zhang J, Tang B, et al. The novel circ_0028171/ miR-218-5p/IKBKB axis promotes osteosarcoma cancer progression. Cancer Cell Int 2020;20:484.

27. Wei W, Ji L, Duan W, et al. CircSAMD4A contributes to cell doxorubicin resistance in osteosarcoma by regulating the miR-218-5p/KLF8 axis. Open Life Sci 2020;15:848-59.

28. Jia G, Wang Y, Yu Y, et al. Long non-coding RNA NR2F1-AS1 facilitates the osteosarcoma cell malignant phenotype via the miR-485-5p/miR-218-5p/BIRC5 axis. Oncol Rep 2020;44:1583-95.

(English Language Editor: A. Kassem)
Cite this article as: Zhang L, Yao M, Ma W, Jiang Y, Wang W. MicroRNA-376b-3p targets RGS1 mRNA to inhibit proliferation, metastasis, and apoptosis in osteosarcoma. Ann Transl Med 2021;9(22):1652. doi: 10.21037/atm-21-4949 